\title{
Análise da Estrutura Interna da Bateria de Avaliação Intelectual de Jovens e Adultos
}

\author{
Solange Muglia Wechsler ${ }^{1}$ \\ Wagner de Lara Machado ${ }^{2}$ \\ Rosangela Kátia Sanches Mazzorana Ribeiro ${ }^{3}$ \\ Adriana Ferreira de Souza ${ }^{4}$ \\ Priscila Schneider ${ }^{5}$ \\ Everley Goetz ${ }^{6}$ \\ Wedja Costa ${ }^{7}$ \\ Carlos Irineu Granja Costa ${ }^{8}$ \\ ${ }^{1}$ Pontifícia Universidade Católica de Campinas, Campinas, SP \\ ${ }^{2}$ Pontifícia Universidade Católica do Rio Grande do Sul, Porto Alegre, RS \\ ${ }^{3}$ Universidade Federal do Mato Grosso, Cuiabá, MT \\ ${ }^{4}$ Faculdades Educatie, Mogi das Cruzes, SP \\ ${ }^{5}$ Universidade do Planalto Catarinense, Lages, SC \\ ${ }^{6}$ Instituto Educacional Superior de Brasilia, Brasilia, DF \\ ${ }^{7}$ Faculdade Unichristus, Fortaleza, CE \\ ${ }^{8}$ Faculdade de Ensino e Cultura do Ceará, Fortaleza, CE
}

\begin{abstract}
Resumo
O objetivo deste estudo foi analisar a estrutura interna e a relação das habilidades cognitivas avaliadas pela Bateria de Avaliação Intelectual de Adultos (BAIAD). A amostra foi composta por 963 indivíduos (615 F, $348 \mathrm{M})$, idades entre 15-64 anos ( $\mathrm{M}=21$, $\mathrm{DP} / \mathrm{SD} / \mathrm{DS}=8)$. A BAID é composta de sete subtestes: sinônimos, antônimos, analogias, pensamento lógico, pensamento viso-espacial, memória visual-auditiva, rapidez de raciocino. A análise de itens permitiu a redução da BAIAD e índices de precisão de 0,99. A análise confirmatória seguiu o modelo CHC (inteligência cristalizada, inteligência fluída e um fator geral - $G$ ) e indicou seu ajuste aos dados. A análise de rede que buscou explorar a relação entre os pares dos indicadores cognitivos e o agrupamento das comunidades apoiou o modelo teórico. Conclui-se que a BAIAD possui evidências de validade e índices de precisão adequados para avaliar a inteligência de adultos.

Palavras-chave: inteligência, avaliação psicológica, testes psicológicos, validade, cognição
\end{abstract}

Analysis of the Internal Structure of the Battery for Assessing Youth and Adults' Intelligence

\begin{abstract}
The purpose of this study was to analyze the internal structure and the relation of the cognitive abilities evaluated by the Battery of Intellectual Assessment of Adults (BAIAD). The sample consisted of 963 subjects (615 F, $348 \mathrm{M}$ ), ages 15-64 years (Median $=21, \mathrm{DP} / \mathrm{SD} / \mathrm{DS}=8)$. The BAIAD is composed of seven tests: synonyms, antonyms, analogies, logical thinking, visual spatial thinking, visual-auditory memory and speed of reasoning. Item analysis allowed the reduction of BAIAD and reached the reliability indexes of 0.99 . The confirmatory analysis followed the $\mathrm{CHC}$ model (crystallized intelligence, fluid intelligence and a general factor $-\mathrm{G}$ ) and indicated its fit to the data. The network analysis which explored the relationship between the pairs of cognitive indicators and the communities grouping supported the theoretical model. We conclude that BAIAD has validity evidences and adequate reliability to assess adults' intelligence.

Keywords: intelligence, psychological assessment, psychological tests, validity, cognition
\end{abstract}

\section{Análisis de la estructura interna de la Batería de Evaluación Intelectual de Jóvenes y Adultos}

\section{Resumen}

El objetivo de este estudio fue analizar la estructura interna y la relación de las habilidades cognitivas evaluadas por la Batería de Evaluación Intelectual de Adultos BAIAD). La muestra fue compuesta por 963 individuos (615 F, $348 \mathrm{M}$ ), con edades entre 15-64 años (Mediana = 21, DP/SD/DS = 8). La BAIAD está compuesta de 7 subtests: sinónimos, antónimos, analogías, pensamiento lógico, pensamiento visoespacial, memoria visual auditiva, rápidez de razonamiento.El análisis de ítems permitió la reducción de la BAIAD e índices de precisión de 0,99. El análisis confirmatorio siguió el modelo CHC (inteligencia cristalizada, inteligencia fluída y un factor general-G) e indicó su ajuste a los datos. El análisis de red que buscó explorar la relación entre los pares de indicadores cognitivos, y el agrupamiento de las comunidades, apoyó el modelo teórico. Se concluye que la BAIAD posee evidencias de validez e índices de precisión adecuados para evaluar la inteligencia de adultos. Palabras clave: inteligencia; evaluación psicólogica; tests psicológicos; validez; cognición 
A medida da inteligência baseada na sua estrutura é alvo de debates desde o século passado. Já em 1904, Charles Spearman demonstrou que era possível medir a inteligência por meio de um fator geral (Fator $G$ ), utilizando a análise fatorial, enquanto que Thurstone, em 1948, defendia que a inteligência deveria ser medida por fatores específicos (Ackerman, 2013; Kranzler \& Floyd, 2013). Posteriormente Cattell (1971) apresentou um modelo hierárquico que concebia dois tipos de inteligência: fluida $(G f)$ e cristalizada $(G c)$, sendo que a $G f$ envolveria capacidades mentais relacionadas com processos lógicos, tais como indução, dedução e o raciocínio não verbal, enquanto que a $G c$ abrangeria os conhecimentos, habilidades e conteúdos decorrentes da experiência educacional e exposição cultural (Bowden, 2013; Kvist \& Gustafsson, 2008). Essa teoria ampliada por Horn (1991), conhecida por Gf-Gc (Horn \& Blankson, 2005) é reconhecida pelo seu grande impacto em vários testes que avaliam a inteligência de forma bidimensional (Abad, Sorrel, Román, \& Colom, 2016; Deary, 2012; Flanagan, McGrew, \& Ortiz, 2000; Nisbett et al., 2012).

Posteriormente, o modelo $G f-G c$ foi questionado por Carroll $(1997,2005)$ e revisado por McGrew (1997, 2009) que realizou uma síntese das formulações e propôs um modelo integrador de Cattell-Horn-Carroll (CHC). No modelo atual do CHC, expandido por Schneider e McGrew (2012), existe o fator $G$ no primeiro estrato, 16 habilidades amplas no segundo estrato, incluindo propostas de inteligência ainda não mensuráveis, tais como as habilidades sensoriais e sinestésicas além de outras 70 habilidades específicas. Atualmente o modelo CHC para explicação da inteligência é o mais aceito internacionalmente, não só por ter influenciado a construção ou revisão de novos testes, mas também por ter demonstrado que seus fatores podem ser encontrados em quase todos os testes e em diferentes culturas (Dombrowski \& Watkins, 2013; Keith \& Reynolds, 2010; Flanagan \& Harrison, 2012; Golay, Reverte, Rossier, Favez, \& Lecert, 2012).

A composição dos fatores intelectuais como proposta pelo $\mathrm{CHC}$ tem sido verificada em várias análises confirmatórias e exploratórias de baterias de inteligência norte-americanas atualmente utilizadas ou revisadas, tais como a Wechsler Intelligence Scale for Children - WISC-IV e WISC-V, o Stanford-Binet IV-SB IV, a Kaufman Assessment Battery for Children - KABC e a Woodcok-Johnson III -WJ-III (Flanagan, Alfonso, \& Reynolds, 2013; Goldstein, 2013; Kaufman, Raiford, \& Coalson, 2016; Lichtenberger \& Kaufman, 2013; Schrank, McGrew, \&
Mather 2014). Por sua vez, a comparação entre as baterias WJ-III e Differential Ability Scale (DAS) pela análise confirmatória indicou o $\mathrm{CHC}$ como sendo o modelo como maior índice de ajuste para descrever os construtos avaliados nesses testes (Sanders, MCintoshk, Rothlisberg, \& Finch, 2013).

A importância do modelo $\mathrm{CHC}$ em testes para a predição do rendimento acadêmico tem sido demonstrada, considerando o fator $G$ como tendo a maior influência, como também das habilidades do segundo estrato como $G f$ e $G c$ no rendimento nas áreas de leitura, escrita e matemática (Beaujean, Parkin, \& Parker, 2014; Caemmer, Maddocks, Keith, \& Reynolds, 2018). Pesquisas em diferentes culturas, tais como a portuguesa (Martins, Gomes, Alves, \& Almeida, 2018) e a sueca (Kvist \& Gustafsson, 2008) têm demonstrado que os fatores de inteligência fluida $(G f)$ e cristalizada $(G c)$ oferecem mais contribuições do que uma visão unidimensional pelo fator $G$, porém ressaltam o impacto de variáveis culturais sobre os resultados nos testes.

De modo alternativo aos modelos fatoriais, alguns estudos investigam a influência recíproca entre habilidades cognitivas, considerando a hipótese do mutualismo (Van der Maas et al., 2006). Segundo essa hipótese, o fator geral do modelo CHC seria explicado pelo histórico de interações recorrentes e benéficas (no sentido de incremento mútuo) entre as habilidades cognitivas ao longo do desenvolvimento. Dito de outra forma, é devido a esse padrão de causalidade mútua que se observa a emergência do fator geral de inteligência, e não o inverso. Essas hipóteses são investigadas por meio de modelos dinâmicos, ou modelos de rede. Nesse modelo, as relações parciais por pares de habilidades cognitivas são representadas graficamente, e sua estrutura é descrita em termos de agrupamentos (comunidades) e influência de habilidades cognitivas no sistema. Desenvolvimentos recentes (Van der Maas, Kan, Marsman, \& Stevenson, 2017) integram a perspectiva fatorial ao modelo mutualista, gerando um framework para compreender e estimar os fatores de nível hierárquico ( $G f, G c$ e $G$ ). No presente estudo, além dos modelos fatoriais correspondentes ao modelo $\mathrm{CHC}$, será investigado o modelo dinâmico das habilidades cognitivas.

\section{Estudos Brasileiros com Testes no Modelo CHC}

Algumas baterias internacionais de inteligência têm sido também investigadas para avaliar a sua adequação no modelo $\mathrm{CHC}$ para a nossa realidade, como aponta a revisão de pesquisas na base SciELO 
e PepPSIC (Alves, Rosa, Silva, \& Sardinha, 2016). Os Estudos foram realizados visando adaptar os testes verbais da bateria Woodcock-Jonson-WJ-III (Wechsler, Vendramini, \& Schelini, 2007) como também verificar as evidências de validade de sua estrutura interna e convergência com outros testes já validados no país, tais como o DFH-III e o WISC- III (Wechsler \& Schelini, 2006; Chiodi e Wechsler, 2009), Porém o estudo de Wechsler et al. (2010) apontou que era necessário a remoção de vários itens, ou sua reformulação, para que uma versão brasileira da WJ-III pudesse ser adaptada e validada para nosso país. Outras baterias internacionais foram também investigadas com amostras brasileiras, como no estudo realizado por Gomes e Borges (2007) com a Factor-Referenced Cognitive Tests, no qual foi confirmada a estrutura do $\mathrm{CHC}$ para 45 testes dessa bateria. Também a estrutura fatorial da Bateria de Fatores Cognitivos de Alta Ordem (BAFACALO) foi analisada pelo modelo CHC com uma amostra brasileira (Gomes, 2010) demonstrando que essa bateria pode avaliar seis habilidades cognitivas amplas deste modelo.

Alguns instrumentos brasileiros têm utilizado a base teórica do modelo CHC para a sua construção. Por exemplo, a Bateria Multidimensional de Inteligência Infantil (Schelini \& Wechsler, 2005), foi desenvolvida a partir desse modelo e teve a estrutura inicial dos seus itens confirmada assim como altos índices de precisão. Também o estudo com amostras brasileiras e portuguesas para investigar a estrutura interna da Bateria de Provas de Raciocínio (BPR-5) demonstrou a existência de um fator único da inteligência, composto pela inteligência fluida, cristalizada, processamento visual, habilidade quantitativa e conhecimento prático de mecânica (Primi \& Almeida, 2000). A comparação das baterias WJ-III e BPR-5 indicou que suas estruturas poderiam ser explicadas por três fatores correlacionados, compostos pela inteligência cristalizada, fluida e processamento visual (Primi, Nakano, \& Wechsler, 2012). Note-se que dentre essas baterias somente a BPR-5 possui normas brasileiras até o momento e está disponível para os psicólogos.

De acordo com a regulamentação do Conselho Federal de Psicologia $(2003,2018)$ e da International Test Commission (2018), é necessário que os testes a serem utilizados dentro de um determinando contexto possuam comprovação empírica de sua validade e precisão. Dentre os testes brasileiros aprovados pelo Conselho Federal de Psicologia como possuindo essas características científicas, que são divulgados na lista do Sistema de Avaliação de Testes Psicológicos - SATEPSI (2018), predominam aqueles que avaliam a inteligência de forma unidimensional (exemplos; Matrizes Progressivas de Raven, Teste Não Verbal de Inteligência- R-1, Teste Não Verbal de Inteligência Geral- Beta III etc.). Com a finalidade de contribuir para avaliação das habilidades cognitivas de adultos, foi proposta a Bateria de Avaliação da Inteligência de Adultos-BAIAD, que avalia as dimensões da inteligência fluida $(G f)$ e cristalizada $(G c)$ por meio de diferentes testes, com administração coletiva. Em estudo inicial com a BAIAD, com seis testes (sinônimos, antônimos, analogias, vocabulário, pensamento lógico e viso-espacial), foram verificadas evidências de sua validade pela sua estrutura interna, confirmando o modelo Gf-Gc (Wechsler et al., 2014).

Considerando a necessidade de mais estudos sobre a validade e a precisão de todos os subtestes que compõem a BAIAD, este estudo visou analisar a sua estrutura modificada, adicionando dois subtestes para a versão anterior (memória visual-auditiva e rapidez de pensamento) e eliminando o subteste de vocabulário por figuras por conter ambiguidades. Nessa nova versão da BAIAD com sete subtestes, visa-se avaliar não somente o nível de ajuste da dificuldade dos itens de cada dos subtestes, mais também investigar se a rede da estrutura das habilidades cognitivas derivadas destes formariam associações com duas dimensões de primeira ordem, $G f$ e $G c$ e uma dimensão comum $(G)$. Dessa maneira, poder-se-ia confirmar a solidez do embasamento teórico e empírico da BAIAD e também suas evidências de validade e precisão.

\section{Método}

\section{Participantes}

A amostra foi composta por 963 indivíduos (615 F, $348 \mathrm{M})$, com idades variando de 15 aos 64 anos $(M$ $=21, D P=8)$. Essas pessoas estavam cursando ou tinham concluído o Ensino Médio (37\%), o Técnico (7\%) e o Superior (56\%) nas mais diferentes áreas, em instituições particulares (53\%) ou públicas (47\%), sendo provenientes dos seguintes estados: São Paulo (64\%), Minas Gerais (6\%), Ceará (10\%), Bahia (5\%), Mato Grosso (12\%) e Santa Catarina (3\%). Essa amostra foi coletada por conveniência nos diferentes locais.

\section{Instrumento}

Bateria de Avaliação da Inteligência de Adultos (BAIAD). Essa bateria revisada é composta por sete subtestes: sinônimos (31 itens), antônimos (31 adjetivos), analogias (30 pares de palavras), pensamento 
lógico (35 itens representando sequência de figuras abstratas), pensamento visuoespacial (30 itens em forma de quebra-cabeças), memória visual-auditiva (30 itens em forma de símbolos, cujo significado é mencionado e deve ser lembrado posteriormente na forma e no significado) e rapidez de pensamento (60 sequências de estímulos com letras e números para serem reconhecidos em tempo limitado).

Os itens da parte verbal da BAIAD foram construídos a partir do dicionário eletrônico Houaiss (2007), e os demais foram elaborados a partir dos estudos de Wechsler com a WJ-III (Wechsler et al., 2010). Em um estudo visando confirmar a estrutura interna da BAIAD, utilizando seis subtestes da sua versão anterior (Wechsler et al., 2014), foi demonstrado que a sua estrutura poderia ser explicada por dois fatores principais: $G c$ ( vocabulário, sinônimos, antônimos) e $G f$ (analogias, pensamento lógico e visuoespacial), ambos correlacionados ( 0,72 para $G c$ e 0,80 para $G f)$ com um fator geral $(G)$. Na nova versão da BAIAD o teste de vocabulário por figuras foi eliminado por se considerar que os desenhos traziam ambiguidades na sua interpretação. Outros subtestes foram adicionados nestes estudo que foram: memória visual-auditiva e rapidez de pensamento.

\section{Procedimento}

Após aprovação do Comitê de Ética (No 15462013.1.0000.5481), foi feito contato com professores universitários de distintas regiões para colaboração na pesquisa. Posteriormente estes também procuraram outras instituições nas suas cidades, que tinham cursos em nível de Ensino Médio como também escolas técnicas, que possuíam alunos com idades iguais ou superiores a 15 anos de idade, para convidá-los a participar da pesquisa. Os participantes assinaram o Termo de Consentimento Livre e Esclarecido antes da pesquisa e realizaram os testes em pequenos grupos, durante $o$ período de duas horas, nas próprias salas de aula ou nos locais de trabalho, com intervalo de 15 minutos de descanso após uma hora de realização dos subtestes. Menores de idade tiveram o Termo de Consentimento assinado por seus pais.

\section{Análise de Dados}

Primeiramente foi feita a análise de itens de seis subtestes da BAIAD (com exceção do teste de Rapidez, cujo tempo limitado não permite testar todos os itens por meio do modelo de Rasch para itens dicotômicos (Boone, Staver, \& Yale, 2014). As medidas de infit e outfit utilizadas como valores variaram de 0,5 a 1,5 . O processo de redução de itens foi realizado por meio dos seguintes passos: a) valores infit-outfit dentro do intervalo definido; b) correlação item-total (ponto-bisserial), acima de 0,30 ; c) investigação da dependência local e dimensionalidade da escala, baseando-se na correlação residual $\left(r_{\mathrm{p}}>0,30\right)$ A seleção final dos itens foi realizada observando-se também a mudança do theta, segundo o modelo Rash, que indicava níveis diferentes de habilidades Outros critérios para seleção de itens como o valor residual de ajuste, correlação item total e 0,5 valor logist foram também utilizados. A precisão foi avaliada utilizando os modelos de Rasch e do alfa de Cronbach.

Posteriormente foi conduzida uma análise fatorial confirmatória com as dimensões da BAIAD de modo a testar o modelo CHC. O modelo compreende uma estrutura hierárquica de dois fatores de primeira ordem $(G f$ e $G c)$ e um fator geral $(G)$. O método de estimação utilizado foi o Maximum Likelihood robusto, por ser próprio para dados com distribuição não normal. Os índices de ajuste considerados foram CFI $(\geq 0,90)$, TLI $(\geq 0,90)$ e RMSEA $(\leq 0,06)$. Utilizou-se os pacotes Lavaan (Rosseel, 2017) e SemPlot (Epskamp, 2017) do software $R$.

Por fim, com a finalidade de investigar as relações diretas entre os indicadores cognitivos, procedeu-se uma análise de rede. A análise de rede utiliza algoritmos regularizados Least Abosolut Shrinkage and Selection Operator-LASSO (Tibshirani, 2011) para obtenção da matriz inversa de variância/covariância ou matriz de precisão. Quando padronizada, essa matriz representa a correlação parcial entre as variáveis investigadas. Para facilitar a visualização das relações entre as variáveis, a matriz de precisão é representada em um sistema gráfico que inclui as variáveis (nodos) e as relações entre elas (linhas). A espessura e coloração das linhas indicam a magnitude e direção da associação, respectivamente. Por fim um algoritmo de localização é utilizado, fazendo com que variáveis conectadas se atraiam e variáveis com pouco ou nenhuma associação sejam repelidas (Fruchterman \& Reingold, 1991). Possíveis agrupamentos foram investigados por meio da uma análise de comunidades, por meio de um algoritmo multinível (Louvain) (Yang, Algesheimer, \& Tessone, 2016). O algoritmo tem por objetivo otimizar a medida de modularidade (proporção entre as conexões intragrupo e entregrupos) de modo a caracterizar um número adequado de subagrupamentos (comunidades) possivelmente existentes no sistema. 
As análises foram conduzidas por meio do pacote qgraph (Espkamp, Cramer, Waldorp, Schmittmann, \& Borsboom, 2012).

\section{Resultados}

A análise dos itens de cada subteste feita pelo modelo Rasch e demonstrou que muitos itens apresentavam misfit ou inadequações devido a uma baixa taxa de sucesso ou até mesmo adivinhações randômicas. As medidas de infit e outfit utilizadas como valores variaram de 0,5 a 1,5 . A seleção final dos itens também foi realizada observando-se a mudança do theta, segundo o modelo Rash, que indicava níveis diferentes de habilidades. Assim sendo, a BAIAD poderia avaliar diferentes níveis de dificuldade para realizar a tarefa.

A Tabela 1 apresenta os itens finais da BAIAD após a sua seleção segundo os critérios já estipulados, assim como o nível de dificuldade de cada um deles. A precisão de cada subescala foi estimada pelo modelo Rash e pelo coeficiente Cronbach.

Conforme pode ser visto na Tabela 1 , os dados indicam que é possível uma considerável redução de itens da BAIAD, segundo os critérios estipulados. A seguinte redução de itens é proposta para cada subteste: Analogias (de 30 para 10), antônimos (de 31 para 14), sinônimos (de 31 para 10), pensamento lógico (de 35 para 15$)$, memória visual-auditiva (de 30 para 12 )

Tabela 1.

Proposta de Reducão dos Itens pelos Valores Infit-Outfit e Precisão pelo Rasch e Alfa

\begin{tabular}{|c|c|c|c|c|c|c|c|c|c|c|c|c|}
\hline \multirow[t]{2}{*}{$\begin{array}{l}\text { Escala } \\
\text { reduzida }\end{array}$} & \multicolumn{2}{|c|}{$\begin{array}{c}\text { Analogias } \\
n=10\end{array}$} & \multicolumn{2}{|c|}{$\begin{array}{c}\text { Antônimos } \\
n=14\end{array}$} & \multicolumn{2}{|c|}{$\begin{array}{l}\text { Lógico } \\
n=15\end{array}$} & \multicolumn{2}{|c|}{$\begin{array}{c}\text { Memória } \\
n=12\end{array}$} & \multicolumn{2}{|c|}{$\begin{array}{l}\text { Sinônimos } \\
N=10\end{array}$} & \multicolumn{2}{|c|}{$\begin{array}{c}\text { Espacial } \\
N=15\end{array}$} \\
\hline & 17 & 3,29 & 9 & 1,88 & 25 & 2,77 & 16 & 4,26 & 3 & 2,08 & 30 & 2,39 \\
\hline \multirow{14}{*}{$\begin{array}{l}\text { Itens e } \\
\text { dificuldades }\end{array}$} & 12 & 1,77 & 19 & 1,36 & 20 & 1,38 & 7 & 2,37 & 13 & 1,44 & 31 & 1,99 \\
\hline & 10 & 1,65 & 11 & 1,18 & 21 & 1,17 & 11 & 2,05 & 10 & 1,08 & 26 & 1,67 \\
\hline & 16 & 0,61 & 20 & 1,00 & 18 & 1,14 & 12 & 1,13 & 17 & 0,90 & 6 & 1,16 \\
\hline & 4 & 0,05 & 16 & 0,61 & 24 & 1,13 & 8 & 0,96 & 7 & 0,46 & 27 & 0,78 \\
\hline & 18 & $-0,65$ & 4 & 0,58 & 22 & 1,01 & 15 & 0,93 & 18 & 0,06 & 28 & 0,78 \\
\hline & 14 & $-1,06$ & 15 & 0,40 & 27 & 0,93 & 10 & 0,80 & 4 & $-0,78$ & 21 & 0,57 \\
\hline & 7 & $-1,44$ & 2 & 0,23 & 23 & 0,84 & 13 & 0,55 & 1 & $-1,13$ & 19 & 0,37 \\
\hline & 8 & $-1,75$ & 14 & $-0,71$ & 31 & 0,84 & 4 & $-1,20$ & 6 & $-1,29$ & 29 & 0,08 \\
\hline & 2 & -2.46 & 17 & $-1,07$ & 30 & 0,43 & 3 & $-2,31$ & 5 & $-2,83$ & 9 & $-0,14$ \\
\hline & & & 6 & $-1,28$ & 16 & $-0,12$ & 2 & $-3,77$ & & & 12 & $-0,82$ \\
\hline & & & 3 & $-1,45$ & 12 & $-1,15$ & 1 & $-5,73$ & & & 5 & $-1,56$ \\
\hline & & & 1 & $-2,73$ & 8 & $-3,07$ & & & & & 4 & $-1,90$ \\
\hline & & & & & 11 & $-3,41$ & & & & & 3 & $-2,37$ \\
\hline & & & & & 7 & -3.90 & & & & & 14 & $-3,01$ \\
\hline \multirow[t]{2}{*}{ Descritivaa } & & $\begin{array}{c}0,00 \\
(1,72)\end{array}$ & & $\begin{array}{c}0,00 \\
(1,29)\end{array}$ & & $\begin{array}{c}0,00 \\
(1,91)\end{array}$ & & $\begin{array}{c}0,00 \\
(2,68)\end{array}$ & & $\begin{array}{c}0,00 \\
(1,42)\end{array}$ & & $\begin{array}{c}0,00 \\
(1,57)\end{array}$ \\
\hline & \multicolumn{2}{|c|}{ Infit $=1,00$} & \multicolumn{2}{|c|}{ Infit $=0,98$} & \multicolumn{2}{|c|}{ Infit $=0,98$} & \multicolumn{2}{|c|}{ Infit $=0,96$} & \multicolumn{2}{|c|}{ Infit $=0,99$} & \multicolumn{2}{|c|}{ Infit $=0,99$} \\
\hline \multirow{4}{*}{$\begin{array}{l}\text { Indices de } \\
\text { ajustea }\end{array}$} & \multicolumn{2}{|c|}{ Outfit $=1,18$} & \multicolumn{2}{|c|}{ Outfit = 1,16} & \multicolumn{2}{|c|}{$\begin{array}{c}(0,23) \\
\text { Outfit }=1,19\end{array}$} & \multicolumn{2}{|c|}{$\begin{array}{c}(0,14) \\
\text { Outfit }=162\end{array}$} & \multirow{2}{*}{\multicolumn{2}{|c|}{$\begin{array}{l}\text { Outfit }=1,02 \\
(0,18)\end{array}$}} & \multicolumn{2}{|c|}{$\begin{array}{c}(0,10) \\
\text { Outfit }=096\end{array}$} \\
\hline & \multicolumn{2}{|c|}{$(0,32)$} & \multicolumn{2}{|c|}{$(0,51)$} & \multicolumn{2}{|c|}{$(0,53)$} & \multicolumn{2}{|c|}{$(1,07)$} & & & \multicolumn{2}{|c|}{$\begin{array}{c}\text { Outfit }=0,96 \\
(0,26)\end{array}$} \\
\hline & \multicolumn{2}{|c|}{$\begin{array}{c}\text { Precisão b }= \\
0,99\end{array}$} & \multicolumn{2}{|c|}{$\begin{array}{c}\text { Precisão }= \\
0,99\end{array}$} & \multicolumn{2}{|c|}{$\begin{array}{c}\text { Precisão }= \\
0,99\end{array}$} & \multicolumn{2}{|c|}{$\begin{array}{c}\text { Precisão }= \\
0,99\end{array}$} & Prec & $\begin{array}{l}\text { áo }= \\
9\end{array}$ & Prec & áo $=$ \\
\hline & Cro & $\begin{array}{l}\mathrm{ich}= \\
3\end{array}$ & Cro & $\begin{array}{l}\mathrm{ach}= \\
0\end{array}$ & Cror & $\begin{array}{l}\text { ach }= \\
2\end{array}$ & Cror & $\begin{array}{l}\mathrm{ach}= \\
1\end{array}$ & Cron & $\begin{array}{l}\text { ach }= \\
4\end{array}$ & Cror & $\begin{array}{l}\mathrm{ch}= \\
1\end{array}$ \\
\hline
\end{tabular}

Nota. ${ }^{a}$ Médias e desvios padrões por dificuldade de itens, valores infit e outfit, ${ }^{\mathrm{b}}$ Precisão estimada pelo modelo Rasch. 
e espacial (de 30 para 15). A seleção desses itens para cada subteste foi baseada no critério de se escolher itens que pudessem abarcar as faixas de baixa dificuldade (para entrada no teste), itens com média dificuldade e itens com alta dificuldade (para discriminação de indivíduos com mais alta capacidade). É digno de nota que, mesmo reduzindo o número de itens de cada subteste para quase a metade, ainda se obtêm a precisão pelo modelo Rash de 0,99 para cada subteste. O alpha de Cronbach, que é outra estimativa de precisão, variou de 0,64 (para o subteste de Sinônimos) até 0,81 (para o subteste de Raciocínio Espacial). Pode-se concluir que os índices de precisão são satisfatórios para a proposta da BAIAD reduzida.

Em seguida a análise fatorial confirmatória (Figura 1) indicou excelente ajuste do modelo teórico aos dados. O modelo hipotetizado compreende dois fatores de primeira ordem explicados por um fator geral, de segunda ordem. O fator de primeira ordem $G c$ - inteligência cristalizada é formado pelos subtestes de analogias, antônimos e sinônimos, enquanto o fator $G f$ - inteligência fluída é formado pelos subtestes de pensamento logico, memória, raciocínio espacial e rapidez. As cargas fatoriais das subescalas nos fatores de primeira ordem foram todas adequadas $(\geq 0,32)$ e estatisticamente diferentes de zero. O fator geral explica ligeiramente melhor a inteligência cristalizada $(0,86)$. $\mathrm{O}$ modelo como um todo apresenta ajuste, obtendo-se os seguintes índices $\chi^{2}=72,01$ (13), CFI $=0,96$, TLI $=$ 0,94, RMSEA $=0,058$.

A seguir, foram analisadas as redes de associações das habilidades cognitivas na BAIAD reduzida para verificar sua adequavam ao modelo teórico $\mathrm{CHC}$. A rede de correlações parciais encontradas entre os subtestes indicam dois grandes grupos, sendo o primeiro composto pelos testes que avaliam $G f$ (rapidez de raciocínio, pensamento lógico, visuoespacial e memória) e o segundo pelos testes que avaliam a Gc (Antônimos, Sinônimos, Analogias). Os índices de ajuste do modelo de rede foram $\chi^{2}=2,66(11), \mathrm{CFI}=0,99$, TLI $=0,99$, RMSEA $=0,001$. A análise das comunidades é condizente com os fatores de primeira ordem $G f$ e $G c$, encontrados no modelo fatorial (Figura 2).

$\mathrm{Na}$ Tabela 2 são apresentadas as correlações parciais entre os subtestes da BAIAD para melhor visualização. Como pode ser observado, todos os subtestes possuem correlações significativas entre si $(p \leq$ $0,01)$, indicando assim a presença de um fator geral $(G)$. Por sua vez, ao analisar os testes que avaliam a $G c$ (Antônimos, Sinônimos, Analogias), é possível verificar

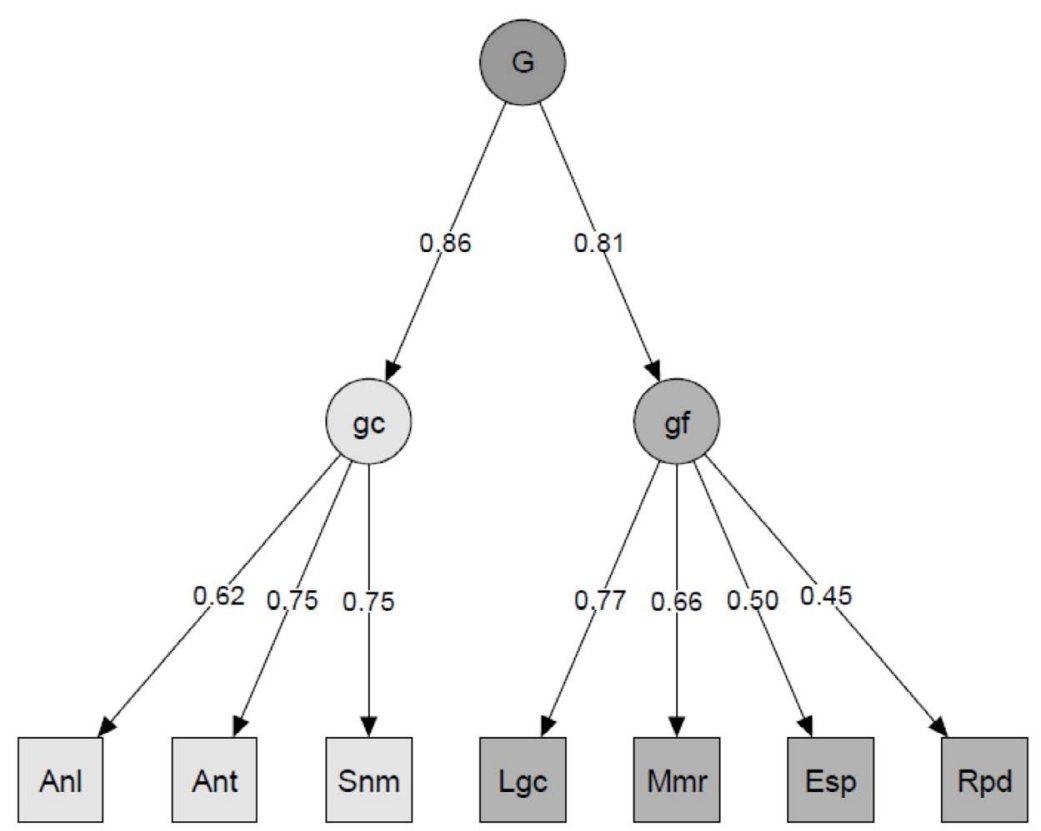

Figura 1. Diagrama da análise fatorial confirmatória. $G=$ fator geral da inteligência, $G c=$ inteligência cristalizada, $G f=$ inteligência fluída, $\mathrm{Anl}=$ analogias, $\mathrm{Ant}=$ antônimos, $\mathrm{Snm}=$ sinônimos, $\mathrm{LGf}=$ pensamento lógico, $\mathrm{Mmr}=$ memória visual auditiva, Esp = pensamento viso- espacial, $\mathrm{Rpd}=$ rapidez de processamento. 


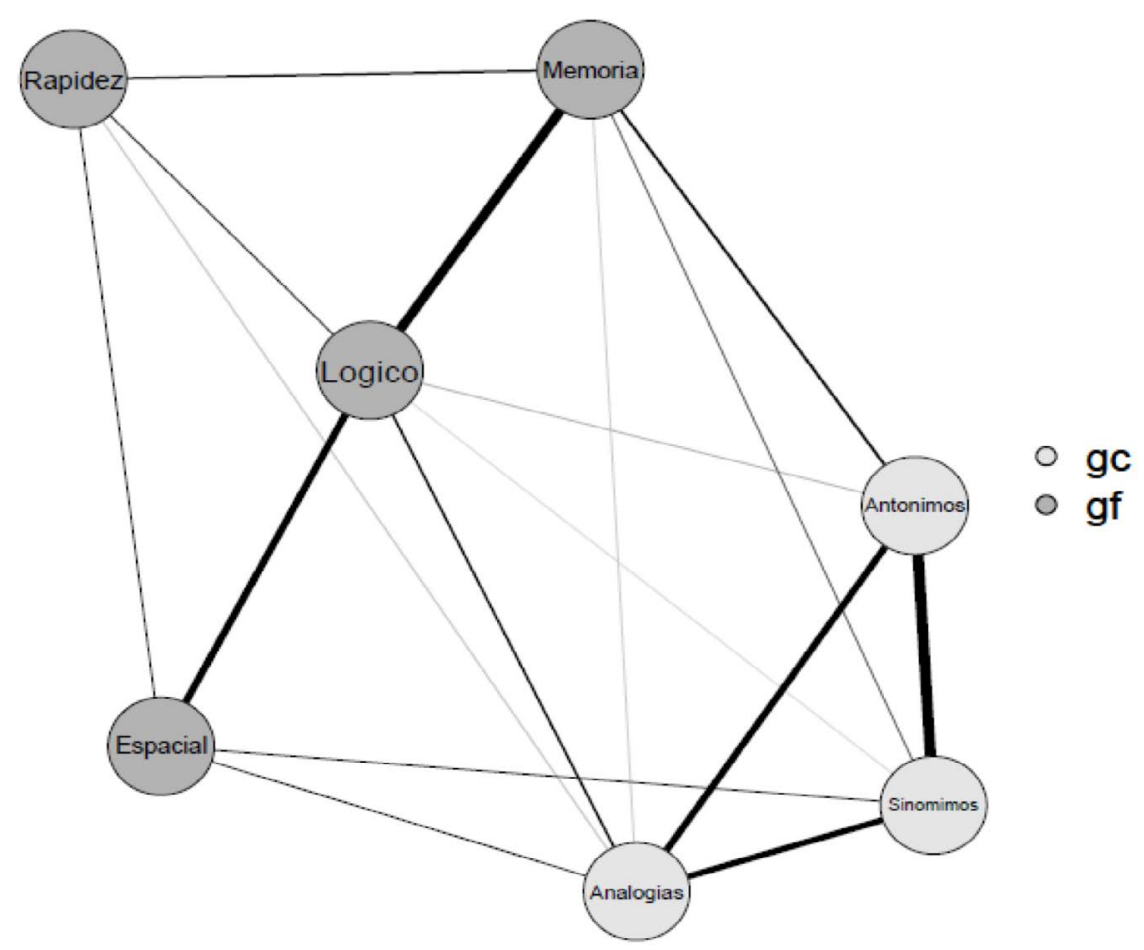

Figura 2. Análise da rede e comunidades entre os subtestes da BAIAD.

que possuem altas correlações significativas entre si $\left(r_{\mathrm{p}}\right.$ $>0,63)$, e o mesmo ocorrendo para os testes que avaliam o Gf (pensamento lógico, visuoespacial e memória visual-auditiva) com correlações significativas $\left(r_{p}>\right.$ 0,50). O teste de Rapidez de Raciocínio também apresenta correlações mais altas com os testes que avaliam inteligência fluida-Gf $\left(r_{p}>0,174\right)$ do que os demais. Conclui-se, portanto, que essas associações refletem o modelo teórico $G f-G c$.

\section{Discussão}

A compreensão da estrutura da inteligência é um dos desafios mais presentes na área da avaliação psicológica. Dentre os modelos atuais mais aceitos para a explicação deste construto encontra-se o CHC (Cattell-Horn-Carol), sintetizado por McGrew (1997, 2000) e, posteriormente, revisado por Schneider e McGrew (2012). Entretanto, embora o modelo CHC esteja

Tabela 2.

Correlaçôes Parciais entre os Subtestes da BALAD

\begin{tabular}{|c|c|c|c|c|c|c|c|}
\hline Teste & Analogias & Antônimos & Sinônimos & Lógico & Memória & Espacial & Rapidez \\
\hline Analogias & --- & $0,635^{* *}$ & $0,628 * *$ & $0,454 * *$ & $0,411 * *$ & $0,405^{* *}$ & $0,268^{* *}$ \\
\hline Antônimos & & --- & $0,695^{* *}$ & $0,424 * *$ & $0,458^{* *}$ & $0,353^{* *}$ & $0,177 * *$ \\
\hline Sinônimos & & & --- & $0,412^{* *}$ & $0,421 * *$ & $0,386^{* *}$ & $0,174 * *$ \\
\hline Lógico & & & & ---- & $0,585^{* *}$ & $0,509 * *$ & $0,326^{* *}$ \\
\hline Memória & & & & & --- & $0,347^{* *}$ & $0,307^{* *}$ \\
\hline Espacial & & & & & & ---- & $0,276^{* *}$ \\
\hline Rapidez & & & & & & & --- \\
\hline
\end{tabular}

Nota. $*_{p} \leq 0,05 ; * * p \leq 0,01$ 
sendo usado internacionalmente para a construção de testes de inteligência, tal fato não ocorre na nossa realidade, pois são escassos os testes que se baseiam nessa proposta conceitual (Primi \& Almeida, 2000; Schelini \& Wechsler, 2005), predominando os testes que avaliam a inteligência de forma unidimensional (Alves, et al., 2016).

Nesse sentido, visando contribuir para a área da avaliação intelectual, foi criada a BAIAD que pretende avaliar a inteligência de jovens e adultos por meio de sete subtestes (sinônimos, antônimos, analogias, pensamento lógico, raciocínio visuoespacial, memória visual-auditiva e rapidez de pensamento). Esta bateria foi construída após vários estudos com a Woodcock-Johnson III (WJ-III) que demonstrou não estar adequada para uso no nosso país devido ao nível de dificuldade existente nos seus itens, principalmente no que concernia à área verbal (Wechsler et al., 2010). Considerando a necessidade de comprovação da validade e precisão dos instrumentos psicológicos, de acordo com as exigências do Conselho Federal de Psicologia (2018) e as recomendações da International Testing Commission (2018), neste estudo, foram investigadas as evidências de validade e precisão da BAIAD.

A estrutura fatorial da BAIAD já tinha sido anteriormente investigada com seis subtestes, com uma amostra menor, no qual foi confirmado que avaliava as dimensões de $G f$ e Gc (Wechsler et al., 2010). Porém ainda faltavam mais outras áreas a serem investigadas, sendo então decidida a inclusão dos subtestes de memória visual-auditiva e de rapidez de processamento. Além disto, não havia sido feito ainda uma análise da sua dificuldade de itens da BAIAD e nem investigadas as evidências de validade e precisão com todos estes subtestes. Neste estudo, além da redução dos itens da BAIAD considerando o seu desajuste e dificuldade, foram testados dois modelos para explicar a sua estrutura interna como também a sua precisão, que foram de análise fatorial confirmatória e de redes

Os resultados obtidos demonstraram que os subtestes da BAIAD se aglomeraram em dois grandes fatores, que explicaram a inteligência fluída-Gf (Rapidez de Raciono, Pensamento Lógico, Visuoespacial e Memória) e inteligência cristalizada-Gc (Antônimos, Sinônimos, Analogias). Assim sendo, os dois modelos de análises utilizados confirmaram o modelo teórico da BAIAD e, consequentemente, apresentaram evidências de sua validade pela sua estrutura interna. Essa estrutura pode ser considerada mais adequada ao modelo teórico $G f-G c$, pois, no estudo anterior com essa bateria
(Wechsler et al., 2014), o subteste de analogias estava pertencendo à inteligência fluida. Pesquisas internacionais, como, por exemplo, em Portugal e na Suécia, também confirmaram a presença da inteligência fluída $(G f)$ e cristalizada $(G c)$ nos seus testes de inteligência (Martin, Alves, \& Almeida, 2016; Kvist \& Gustafsson, 2008), porém indicaram que as variáveis de cada cultura devem ser consideradas no seu impacto sobre o rendimento nos testes.

Também foi observado que é possível diminuir o tempo de administração desta bateria eliminando itens com desajustes. Tal redução de tempo traz ganhos práticos para o psicólogo brasileiro, que dispõe de poucos instrumentos que avaliem diversas áreas do funcionamento intelectual do adulto, porém com maior tempo de administração, como, por exemplo, o Wechsler Intelligence for Adults - WAIS-III ( adaptada por Nascimento, 2015). Por sua vez, a análise da precisão estimada pelos modelos Rasch como também pelo alfa de Cronbach alcançaram resultados satisfatórios. Pode-se concluir, portanto, que a BAIAD atende aos critérios científicos de precisão para instrumentos psicológicos.

As limitações da pesquisa também devem ser ressaltadas. A necessidade de ampliação da coleta de dados para outras regiões do país é uma das grandes barreiras que existiu neste estudo, principalmente no que se refere às regiões Norte e Nordeste. Do mesmo modo ainda são necessários estudos que utilizem critérios externos para verificação da validade da BAIAD, comparando seus dados com testes já validados no país que avaliem construtos específicos de cada um dos seus subtestes. Futuramente, também deverão ser traçadas normas para o uso da BAIAD, em formato reduzido, a fim de calcular a eficiência desta forma de aplicação e poderem ser traçados parâmetros de comparação.

A contribuição da BAIAD pode ser vista sob diferentes ângulos. Os seus resultados poderão apontar áreas fortes e fracas do funcionamento intelectual de um indivíduo. Dessa maneira, poderá fornecer um diagnóstico diferencial de habilidades que podem ser trabalhadas em programas de estimulação do potencial cognitivo. Também fornece elementos importantes para avaliar áreas de melhor funcionamento individual, ao contrário de medidas individualizadas que só permitem identificar uma área cognitiva ou que geram um resultado total da inteligência sem discriminar potenciais. Finalmente, a administração coletiva dos subtestes da BAIAD traz implicações para o uso mais generalizado em situações de grupo, tais como orientação 
vocacional ou seleção profissional, trazendo assim uma grande contribuição para essas áreas de atuação.

\section{Referências}

Abad, F. J.,Sorrel, M. A.,Román, F. J. \& Colom, R. (2016) The relationships between WAIS-IV factor index scores and educational level: A bifactor model approach. Psychological Assessment, 28(8), 9871000. doi: $10.1037 /$ pas0000228

Ackerman, P. (2013). Assessment of intellectual functioning in adults. Em K. F. Geinsinger (Eds.), APA handbook of testing and assessment in Psychology. Testing and Assessment in Clinical and Counseling Psychology (pp.119-132). Washington, DC: American Psychological Association.

Alves, I. C. B., Rosa, H. R., Silva, M. A., \& Sardinha, L. S. (2016). Avaliação da inteligência: Revisão da literatura de 2005 a 2014. Avaliação Psicológica, 15 (n. esp), 89-97. doi: 10.15689/ap.2016.15ee.09

Beaujean, A. A., Parkin, J., \& Parker, S. (2014). Comparing Cattell-Horn-Carroll factor models: Differences between bifactor and higher order factor models in predicting language achievement. Psychological Assessment, 26(3), 789-805. doi: 10.1037/a0036745

Boone, W. J., Staver, J. R., \& Yale, M. S. (2014). Rasch Analysis in the Human Sciences.Dordrecht: Springer. doi: 10.1007/978-94-007-6857-4

Bowden, S. C. (2013). Theoretical convergence in assessment of cognition. Journal of Psychoeducational Assessment, 31(2), 148-156. doi:10.1177/0734282913478035

Caemmer, J. M., Maddocks, D. L. S., Keith, T. Z., \& Reynolds, M. R. (2018).Effects of cognitive abilities on child and youth academic achievement: Evidence from the WISC-V and WIAT-III. Intelligence, 68, 6-20. doi: 10.1016/j.intell.2018.02.005

Cattell, R. B. (1971). Abilities: Their structure, growth and action. Boston: Houghtoun Mifflin.

Carroll, J. (1997). The three-stratum theory of cognitive abilities. Em D. P. Flanagan, J. L. Genshaft \& P. L. Harrison (Eds.), Contemporary intellectual assessment: Theories, tests and issues ( pp. 122-130). New York: Guilford Press.

Carroll, J. B. (2005). The three-stratum theory of cognitive abilities. Em D. P. Flanagan \& P. L. Harrison
(Eds). Contemporary intellectual assessment: Theories, tests and issues (2nd.ed.), (pp 69-76). New York: The Guilford Press.

Chiodi, M. G., \& Wechsler, S. M. (2009). Escala de inteligência WISC-III e Bateria de Habilidades Cognitivas Woodcock-Johnson III: Comparação de instrumentos. Avaliação Psicológica, 8, 313-324.

Conselho Federal de Psicologia (2003). Resolução CFP 002/ 2003. Brasília: Conselho Federal de Psicologia.

Conselho Federal de Psicologia (2018). Resolução N. 9 de 25 de abril de 2018.

Brasília: Conselho Federal de Psicologia.

Deary, I. (2012) Intelligence. Annual Review of Psychology, 63(1), 453-482. doi: 10.1146/ annurev-psych-120710-100353

Dombrowski, S. C., \& Watkins, M. W. (2013). Exploratory and higher order factor analysis of the WJ-III full test battery: A school-aged analysis. Psychological Assessment, 25(2), 442-455. doi: 10.1037/a0031335

Epskamp, S. (2017). Package SemPlot. Version 1.1. Repository CRAN. Recuperado de https://cran.r-project.org/web/packages/semPlot/semPlot. pdf

Espkamp, S., Cramer, A. O. J.,Waldorp, L. J., Schmittmann, V. D., \& Borsboom, D. (2012). Qgraph: Network visualizations of relationships in psychometric data. Jornal of Statistical Software, 48(4), 1-18.

Flanagan, D. P., Alfonso, V. C., \& Reynolds, M. R. (2013). Broad and narrow CHC abilities measured and not measured by the Wechsler Scales: Moving beyond within-battery factor analysis. Journal of Psychoeducational Assessment, 31(2), 202-223. doi: http://dx.doi.org/10.1177/0734282913478047

Flanagan, D. P., McGrew, K .S., \& Ortiz, S. O. (2000). The Wechsler intelligence scales and Gf-Gf theory: $A$ contemporary approach to interpretation. Boston: Allyn and Bacon.

Flanagan, D. P., \& Harrison, P. L. (2012) . Contemporary Intellectual Assessment, Second Edition: Theories, Tests, and Issues ( $3^{\text {rd }}$ ed.). Guilford Press: New York.

Fruchterman, T. M. J., \& Reingold, E. M. (1991). Graph drawing by force-directed placement. Software-practicce and Experience, 21(11), 1129-1164. 
Golay, P., Reverte, I., Rossier, J., Favez, N., \& Lecert, T. (2012). Further insights on the French WISC-IV: Factor structure through Bayesian structural equation modeling. Psychological Assessment, 25, 496-508. doi:10.1037/a0030676

Goldstein, S. (2013). The science of intelligence testing: Commentary on the evolving nature of interpretations of the Wechsler scales. Journal of Psychoeducational Assessment, 31(2), 132-137. doi: 10.1177/0734282913478033

Gomes, C. M. A, \& Borges, O. N. (2007). Validação do modelo de inteligência de Carroll em uma amostra brasileira. Avaliação Psicológica, 6(2), pp. 167-17.

Gomes, C. M. A (2010). Estrutura fatorial da bateria de fatores cognitivos de alta-ordem (BAFACALO). Avaliação Psicológica, 9(3), pp. 449-459.

Horn, J. L. (1991). Measuring of intellectual capabilities: A review of theory. Em K. S McGrew, J. K. Werder \& R. W. Woodcock (Eds.). WJ-R technical manual. Chicago: Riverside Publisher.

Horn, J. L., \& Blankson, N. (2005). Foundations for better understanding of cognitive abilities. Em In D. P. Flanagan \& P. L. Harrison (Eds).Contemporary Intellectual Assessment, Theories, Tests, and Issues. $2^{\text {nd }}$ ed. (pp.41-68). New York: Guilford Press.

Houaiss. A. (2007). Dicionário da Lingua Portuguesa. CD - Versão eletrônica 2.0. Rio de Janeiro, RF: Instituto Antonio Houaiss.

International Test Commission (2018). ITC Guidelines on Test Use.V1.2. Recuperado de https:/ /www.intestcom.org/page/5

Kaufman, A. S., Raiford, S. E., \& Coalson, D. L. (2016). Intelligent Testing with the

WISC-V. New Jersey: John Wiley and Sons.

Keith, T., \& Reynolds, M. (2010). Cattell-Horn-Carroll abilities and cognitive tests: What we learned from 20 years of research. Psychology in the Schools, 635650. doi: 10.1002/pits.20496

Kranzler, J. H., \& Floyd, R. G. (2013). Assessing intelligence in children and adolescents. New York: The Guilford Press

Kvist, A. V. K., \& Gustafsson, J. E. (2008). The relation between fluid intelligence and the general factor as a function of cultural background: A test of Cattell's Investment theory. Intelligence, 36, 422-436.
Lichtenberger, E. O., \& Kaufman, A. S. (2013). Essentials of WAIS-IV Assessment. New Jersey. John Wiley and Sons.

Martins, A., Gomes, C. M. A., Alves, A. F., \& Almeida, L. S. (2018). The structure of intelligence in childhood: Age and sócio-familar impact on cognitive differentiation. Psychological Reports, 121(1), 79-92.

McGrew, K. S. (1997). Analysis of the major intelligence batteries according toa proposed comprehensive Gf-Gf framework. Em D. P. Flanagan, J. L.Genshaft \& P. L. Harrison (Eds.), Contemporary intellectual assessment:Theories, tests, and issues (pp. 151179). New York: Guilford.

McGrew K. S. (2009). CHC theory and the human cognitive abilities project: Standing on the shoulders of the giants of psychometric intelligence research. Intelligence, 37(1),1-10. doi: 10.1016/j. intell.2008.08.004

Nascimento, E. (2015). WAIS III- Escala de inteligência Wechslerpara Adultos

Adaptação brasileira. São Paulo: Casa do Psicólogo.

Nisbett, R. E., Aronson, J., Blair, C., Dickens, W., Flynn, J., ... Halpern, D. F. (2012). Intelligence: New findings and theoretical developments. American Psychologist, 67(2), 130-159. doi: $10.1037 / \mathrm{a} 0026699$

Primi, R., \& Almeida, L. S. (2000). Estudo de validação da Bateria de Provas de Raciocínio (BPR-5). Psicologia: Teoria e Pesquisa,16,(2),165-173.

Primi, R., Nakano, T. C., \& Wechsler, S. M. (2012). Cross-battery analysis of the Battery of Reasoning Abilities (BPR-5) with the Woodcock-Johnson Cognitive Tests (WJ-III). Temas em Psicologia, 20, 121-132.

Rosseel, Y. ( 2017). Structural Equation Modeling: Models, software and stories. Recuperado de http://schd.ws/hosted_files/user2017/62/rosseel_user2017.pdf

Sanders, S., MCintoshk D., D. M., Rothlisberg, B. A., \& Finch, H, (2013). Joint confirmatory factor analysis of the Differential Ability scales and the Woodcock-johnson Tests of Cognitive abilities-third edition. Psychology in the Schools, 44(2). doi: 10.1002/ pits. 2021 
Sistema de Avaliação de Testes Psicológicos- SATEPSI (2018). Testes favoráveis. Recuperado de http://satepsi.cfp.org.br/listaTesteFavoravel.cfm

Schelini, P. W., \& Wechsler, S. (2005). Bateria Multidimensional de Inteligência

Infantil: desenvolvimento de instrumento. Psico-USF, 10(2), 129-139.

Schneider, W. J., \& McGrew, K. (2012). The CattellHorn-Carroll model of intelligence. Em D. Flanagan \& P. Harrison(Eds.), Contemporary Intellectual Assessment: Theories, Tests, and Issues (3rd ed.) (p. 99-144). New York: Guilford.

Schrank, F. A., McGrew, K. S., Mather, N., (2014). Woodcock-Johnson IV Tests of Cognitive Abilities. Riverside: Rolling Meadows, IL.

Tibshirani, R. (2011). Regression shrinkage and selection via the lasso: A retrospective. Royal Statistics Society, 73(3), 273-282.

Van der Maas, H. L. J., Dolan, C. V., Grasman, R. P. P. P., Wicherts, J. M., Huizenga, H. M., \& Raijmakers, M. E. J. (2006). A dynamical model of general intelligence: The positive manifold of intelligence by mutualism. Psychological Review, 113(4), 842-861. doi: 10.1037/0033-295X.113.4.842

Van der Maas, H. L. J., Kan, K., Marsman, M., \& Stevenson C. E. (2017). Network models for cognitive development and intelligence. Journal of Intelligence, 5 (16). doi: 10.3390/jintelligence5020016
Wechsler, S. M., Nunes, C., Schelini, P. W., Pasian, S. R., Moretti, L, Anache, A.. (2010). Brazilian adaptation of the Woodcock-III tests. School Psychology International, 31(4), 409-421

Wechsler, S. M., \& Schelini, P. W. (2006). Bateria de Habilidades Cognitivas Woodcock-Johnson III: Validade de construto. Psicologia, Teoria e Pesquisa, 22, 287- 295.

Wechsler, S. M., Vendramini, C. M. M., \& Schelini, P. W. (2007). Adaptação brasileira dos testes verbais da Bateria Woodcok-Johnson III. Revista Interamericana de Psicologia/Interamerican Journal of Psychology. 41(3), 285-294.

Wechsler, S. M., Vendramini, C. M. M., Schelini, P. W., Lourençoni, M. A., Souza, A. A. F., \& Mundim, M. C. B. (2014). Factorial structure of the Brazilian Adult Intelligence Battery. Psychology \& Neuroscience, 7(4), 559-566

doi: 10.3922/j.psns.2014.4.15

Yang, Z., Algesheimer, R., \& Tessone, C. (2016). A comparative analysis of community detection algorithm on artificial networks. Scientific Reports, 6(30750). doi: 10.1038/srep30750

Recebido em: 12/09/2018

Reformulado em: 05/12/2018

Aprovado em: 12/12/2018 
Nota dos autores:

Agradecimentos: Os autores agradecem aos financiamentos em diferentes momentos desta pesquisa ao Conselho Nacional de Desenvolvimento Técnico e Científico-CNPQ e à Fundação de Amparo a Pesquisa do Estado de São Paulo-FAPESP

Sobre os autores:

Solange Muglia Wechsler é psicóloga, doutora na University of Georgia (EUA), pós-doutora no Torrance Center of Creative Studies, professora do curso de pós-graduação em Psicologia da PUC-Campinas e membro do conselho executivo da International Testing Commission.

ORCID: http://orcid.org/0000-0002-9757-9113

E-mail:wechsler@lexxa.com.br

Wagner de Lara Machado é psicólogo, doutor e pós-doutor na Universidade Federal do Rio Grande do Sul, professor da Pontifícia Universidade Católica do Rio Grande do Sul e membro da diretoria da Associação de Psicologia Positiva e da Associação Brasileira de Criatividade e Inovação.

ORCID: https://orcid.org/0000-0001-5555-5116

E-mail:wag.lm.psico@gmail.com

Rosangela Kátia Sanches Mazzorana Ribeiro é psicóloga, mestre na PUC-RS, doutora na UFSP, professora adjunta do Curso de Psicologia e do Programa de Pós-Graduação em Psicologia da Universidade Federal de Mato Grosso e membro da Internacional Society of the Rorschach and Projective Methods, membro da Associação Brasileira de Rorschach e Métodos Projetivos.

ORCID: https://orcid.org/0000-0003-4072-1091

Adriana Ferreira de Souza é psicóloga, mestre e doutora pela Pontifícia Universidade Católica de Campinas, professora do curso de Psicologia da Universidade de Mogi das Cruzes e do programa de Mestrado Profissional e Psicogerontologia do Instituo Educatie de Ensino e Pesquisa.

ORCID: https://orcid.org/0000-0002-4756-5768

Priscila Schneider é mestre em Psicologia (UFSC), especialista em Gerontologia e em Metodologias Ativas em Ensino e Aprendizagem, professora da Universidade do Planalto Catarinense e psicóloga (UFSC) do Centro Especializado em Reabilitação - CER II da UNIPLAC.

ORCID: https://orcid.org/0000-0003-1561-3021

Everley Goetz é psicóloga, mestre e doutora pela Universidade Federal de Santa Catarian, professora do Instituto Superior de Goiás (IESGO) e coordenadora do curso de Psicologia do Instituto Superior de Ensino de Brasil (IESB - campus Sul e Oeste).

ORCID: https://orcid.org/0000-0002-1018-4830

Wedja Josefa Granja Costa é psicóloga, doutora em Gestão pela Universidade de Trás-os-Montes e Alto Douro (Portugal) e professora do Curso de Pós-Graduação de Avaliação Psicológica na Universidade Christus em Fortaleza e na Leão Sampaio em Juazeiro.

ORCID: https://orcid.org/0000-0002-0974-5581

Carlos Irineu Granja Costa é psicólogo, mestre em Administração pela Universidade de Fortaleza e diretor do Instituto Wedja de Socionomia.

ORCID: https://orcid.org/0000-0002-6617-2329

Contato com os autores:

Av. John Boyd Dunlop, S/N, Jardim Londres

Campinas-SP, Brasil

CEP: 13034-685 